\title{
Drosophila parasitoid wasps bears a distinct DNA transposon profile
}

\author{
Alexandre Freitas da Silva ${ }^{1 \dagger}$, Filipe Zimmer Dezordi ${ }^{1 \dagger}$, Elgion Lucio Silva Loreto ${ }^{2}$ and Gabriel Luz Wallau ${ }^{3^{*}}$ (D)
}

\begin{abstract}
Background: The majority of Eukaryotic genomes are composed of a small portion of stable (non-mobile) genes and a large fraction of parasitic mobile elements such as transposable elements and endogenous viruses: the Mobilome. Such important component of many genomes are normally underscored in genomic analysis and detailed characterized mobilomes only exists for model species. In this study, we used a combination of de novo and homology approaches to characterize the Mobilome of two non-model parasitoid wasp species.

Results: The different methodologies employed for TE characterization recovered TEs with different features as TE consensus number and size. Moreover, some TEs were detected only by one or few methodologies. RepeatExplorer and dnaPipeTE estimated a low TE content of 5.86 and $4.57 \%$ for Braconidae wasp and 5.22\% and $7.42 \%$ for L. boulardi species, respectively. Both mobilomes are composed by a miscellaneous of ancient and recent elements. Braconidae wasps presented a large diversity of Maverick/Polintons Class II TEs while other TE superfamilies were more equally diverse in both species. Phylogenetic analysis of reconstructed elements showed that vertical transfer is the main mode of transmission.

Conclusion: Different methodologies should be used complementarity in order to achieve better mobilome characterization. Both wasps genomes have one of the lower mobilome estimates among all Hymenoptera genomes studied so far and presented a higher proportion of Class II than Class I TEs. The large majority of superfamilies analyzed phylogenetically showed that the elements are being inherited by vertical transfer. Overall, we achieved a deep characterization of the mobilome in two non-model parasitoid wasps improving our understanding of their evolution.
\end{abstract}

Keywords: Transposable elements, Repetitive elements, Evolution, Vertical transfer, Active mobilome

\section{Background}

Transposable elements (TEs) are genetic elements discovered originally in maize (Zea mays) by Barbara McClintock [1]. Since then, geneticists have found that they are ubiquitous and can account for a large fraction of some genomes, such as 50 and $85 \%$ of primates and maize genomes [2]. Together, TEs and endogenous viruses compose the eukaryotic genome mobilome and such genomic parasites hijack the host molecular machinery for their own replication [3]. New copies of those parasites may generate deleterious mutations to the host genome but most of the

\footnotetext{
* Correspondence: gabriel.wallau@cpqam.fiocruz.br

${ }^{+}$Alexandre Freitas da Silva and Filipe Zimmer Dezordi contributed equally to this work.

${ }^{3}$ Departamento de Entomologia, Instituto Aggeu Magalhães (IAM), Fundação Oswaldo Cruz (FIOCRUZ/PE), Recife, Pernambuco, Brazil

Full list of author information is available at the end of the article
}

observed insertions are probably slightly deleterious or neutral to the organism [4]. However, accumulating evidence shows that the TEs can also generate genetic variability that can be co-opted for a new host functions [5-7].

A robust characterization of the mobilome in several species is essential to evaluate evolutionary interplay between between genomic parasites and host species [8]. Well-characterized mobilomes are restricted to model organisms while it remains largely unexplored in non-model species. However, genome-wide non-model organisms studies are revealing different genomic parasites/host dynamics which differ substantially from the most studied ones. It highlights that our current view of genome and mobilome evolution focusing on few well-studied species is likely biased.

A large number of bioinformatic softwares are available for genome-wide TEs characterization, but most of them 
require a complete or draft assembly genome as input [911]. However, depending on the fragmentary nature of draft assemblies TE characterization can be largely compromised [12]. In order to characterize the mobilome in the absence of a genome assembly new approaches were developed [13] and further improved allowing the characterization of the mobilome directly from a large number of small sequenced reads $[10,14]$. A growing number of studies are characterizing the mobiome of non-model organisms using these approaches [15], such as the grasshopper (Gomphocerus sibiricus) [16], killifish (Austrolebias charrua) [17], the Asian Tiger Mosquito (Aedes albopictus) [18] and the repetitive landscape of different Musaceae species [19].

Insect genomes have a huge variability in TE diversity and content but overall a direct relation of TE content and genome size can be seen with a higher abundance of Class I over Class II TEs [20, 21]. However, when analyzing the main eukaryotic taxa (plants, fungi and some other specific animal taxa) no clear pattern emerges showing that TE content and diversity only correlates with the evolutionary history of the studied species [22, 23]. It highlights the need for better and robust mobilome characterization in organisms from a more diverse set of taxa in order to test the associations between TE content/diversity and genome size [23].

Parasitoid wasps are insects from the Hymenoptera order particularly known due to their specific relationship with several arthropod species. Females of parasitoid wasp species inject venom and deposit eggs on or inside of their hosts [24]. The venom is composed of several molecules along with viral-like particles (VLPs), which are responsible for the inhibition of the host's immunological system. VLPs also can act as a vector of DNA fragments between wasps and their hosts [25-27]. Wasp genomes are underrepresented in genomic surveys and so far only four genomes are available: three closely related Nasonia genomes (Pteromalidae family) and the Fopius arisanus genome (Braconidae family) [28]. However, only Nasonia vitripennis genome has a reported mobilome showing one of the highest high TE content (28.8\%) among Hymenopteran genomes [24].

This study aimed to describe and compare the mobilome of two Drosophila parasitoid wasps: Leptopilina boulardi (Figitidae family) and a wasp from the Aphidius genus (Braconidae family) and evaluate their evolutionary history. We demonstrated that these genomes have a large diversity of TE superfamilies with ancient and recent TEs. Moreover, phylogenetic reconstruction of each superfamily showed that the majority of elements identified were transmitted through vertical transfer.

\section{Methods}

\section{Samples and DNA sequencing}

Wasp specimens were sampled at Santa Maria City, latitude 34.95303 and longitude -120.43572 parasitizing
Drosophila flies - Leptopilina boulardi parasites several Drosophila species while Braconidae species is a restricted parasite of Drosophila species from the flavopilosa group - a highly specialized species group that uses flowers as unique breeding sites [29-31]. It is important to emphasize that we tried to identify the Braconidae wasp at the lowest taxonomic level possible, but after contacting specialized taxonomists it was not possible to reach species identification. Therefore, we will call this species from now on as braconid or Braconidae wasp although it is closely related to the Aphidius genus in a previous COI analysis [29-31]). Genomic DNA was prepared with TruSeq DNA HT Sample Prep Kit (Illumina) according to the manufacturer's instructions and sequenced in a Solexa-Illumina HiSeq 2000 New Generation Sequencing (NGS) device using a single-end approach of read length of $100 \mathrm{bp}$ [29].

\section{Transposable elements characterization}

Characterization and evolutionary study of TEs from wasps were performed following the pipeline in (Additional file 1).

We have used two complementary approaches to characterize the mobilome from raw Illumina reads: I) Raw reads were used as input for RepeatExplorer (RE) analysis pipeline with default parameters. Wasp datasets were independently analyzed. Raw reads clustering were performed using an all-to-all similarity comparison which builds a graph relative to each group of a repetitive element [14]. RepeatExplorer annotated the reads of each assembled cluster using RepeatMasker (http:// www.repeatmasker.org) [15] against the Repbase database [32]. Following, we sought to characterize the top clusters (clusters that represent more than $0.01 \%$ of the reads used) having the majority of the reads with BLAST hit to a known Repbase TE. Resulting top clusters contigs were then reassembled using CAP3 [33] with the following parameters (-a 20 -b 20 -c 12 -d 200 -e 30 -f 20 -g 6 -m 2 -n 5 -p 80 -r 1 -s 900 -t 300 -u 3 -v 2 -o 40) as used by others [17, 34] (Additional file 1). II) dnaPipeTE [10] were run with two Trinity iteractions and variable amount of reads to evaluate its performance and find the best parameter set. Final parameters are as follow: -sample_size $14,000,000$ (the maximum number of reads allowed using two trinity iteractions, considering that we have around 28Mi reads for each wasp species), -sample_number 2 and -RM_t 0.5. Those two approaches were used to estimate the proportion of each TE class and superfamilies in the two genomes (Additional file 1).

Additionally, we performed one de novo characterization using RepeatScout 1.0.5 (RS) [35] using the original assembly obtained from Ortiz et al. 2015. Finally, we clustered three TE libraries generated by these programs plus the Ortiz et al. 2015 TE library, characterized by BLASTn 
against Repbase, using CD-HIT-EST 4.6 [36] with parameters (-c 0.8 -G 0 -aS 0.8 -g 1 -M 50000 -T 8 -n 5) to generate the final TE dataset for each wasp (Additional file 1).

\section{Evolutionary analysis}

In order to reconstruct the evolutionary history of each TE superfamily reliably one need to obtain the largest coding region of each consensus assembled. Hence, we performed open read frame (ORFs) searches only in the RepeatExplorer TE dataset (largest consensus obtained) using getorf (> 100aa) implemented in EMBOSS package [37]. CD-SEARCH was used to identify TE conserved domains [38] against the CDD database (CDD v3.1650,369 PSSMs). All analysis after RepeatExplorer clusterization was performed independently in the respective standalone softwares (CAP3 and getorf). After that we recovered homologous sequences using three different strategies. I - a homology search using protein sequences with known TE domains against Repbase using CENSOR [39] with default parameters. Sequences with the best scores were retrieved for each search. II - BLASTp searches against non-redundant protein sequence (nr) NCBI database with default parameters. Up to 50 protein sequences with the best scores were retained after removing specie-specific redundancies. III - Literature review relative to each superfamily studied and curated TE sequences were retrieved. The Repbase, Literature and BLASTp retrieved sequences were analyzed through getorf [40] to recover their potential coding regions (Additional file 1).

Alignment of each superfamily was performed using protein sequences with more than 100 amino acids using MAFFT [41] and edited manually to remove highly variable regions. Such an approach was taken instead of automatic software due to the high variability in the ORFs size and amino acid composition in TE protein sequences. For instance, even loosening GBlocks parameters almost all alignments sites were removed in the final alignment of some superfamilies (data not shown). Phylogenetic trees were built through PhyML [42] and protein substitution models were evaluated through Smart Model Selection implemented in the same PhyML server (http://www.atgc-montpellier.fr/phyml/). Trees were visualized and colored using FigTree 1.4.3 [43] and iTOL web server [44].

\section{Sequence similarity}

A sequence similarity analysis, one per superfamily, was performed with the MEGA 7 software [45], to evaluate possible horizontal transposon transfer events and characterize monophyletic groups in the phylogenetic trees. Additionally, we also performed Kimura two parameters (K2P) distance analysis between each read and the consensus generated for each contig in order to estimate the relative age of the family inside each genome.
Only contigs assembled with RE plus CAP3 and used in the phylogenetic analysis were included due to their higher average size and better recovery of complete or almost complete TE coding regions. We first obtained the ACE assembly file where all reads were aligned to the multiple contigs obtained from each cluster, then we extracted a multiple sequence alignment of all reads mapped against the contigs that presented the ORF with the TE conserved domain. Then such MSA was given as input to MEGA 7 to estimate K2P distance.

\section{Results}

\section{TEs characterization by different softwares TEs detection}

Overall TEs detected by the different softwares differed in number varying from 74 to 10,267 in braconid wasp and 105 to 12,796 in $L$. boulardi and average size varying from 113 to 601 in the braconid wasp and from 105 to 672 in L. boulardi genome (Additional file 2).

Graph-based clustering analysis in RepeatExplorer yielded 661.605 and 787.848 clusters from each wasp respectively. Considering only top clusters, 313 and 516 top clusters were retained from braconid and L. boulardi wasps. Eighty-one top clusters from Braconidae wasp and 54 from $L$. boulardi showed homology with TEs sequences from Repbase, representing respectively 5.86\% and 5.22\% of wasp genomes (Additional file 3), with Class II TEs being the most abundant in both species. A total of 18 and 15 TEs superfamilies were found in Braconidae and $L$. boulardi wasps, respectively (Additional file 3).

TEs assembly performed with dnaPipeTE yielded 10,267 and 12,796 annotated elements from Braconidae and $L$. boulardi species representing 4.57 and $7.42 \%$ of each genome respectively (Fig. $1 \mathrm{a}$ and b). Class II TEs were the most abundant in both wasps genomes with 2.73 and $4.79 \%$ of the estimated TE content while Class I correspond to 1.84 and $2.63 \%$ of Braconidae and $L$. boulardi genomes respectively (Fig. $1 \mathrm{a}$ and b). A total of 29 and 36 TEs superfamilies were found in Braconidae and L. boulardi genomes (Additional file 3).

In the Braconidae wasp all 18 superfamilies detected in the RE top clusters were also detected by dnaPipeTE while in L. boulardi 14 out of 15 TE superfamilies detected in the RE top clusters were also recovered by dnaPipeTE ( $L O A$ superfamily was detected only through RE analysis) (Additional file 3). In addition, 11 and 21 superfamilies were only detected by dnaPipeTE in the Braconidae and L. boulardi genomes respectively. Such differences were expected based on the analysis of only top cluster in the RE analysis.

\section{Final consensus reconstructed by each strategy}

In order to generate a final TE library combining all methodologies used to characterize the wasps mobilome 

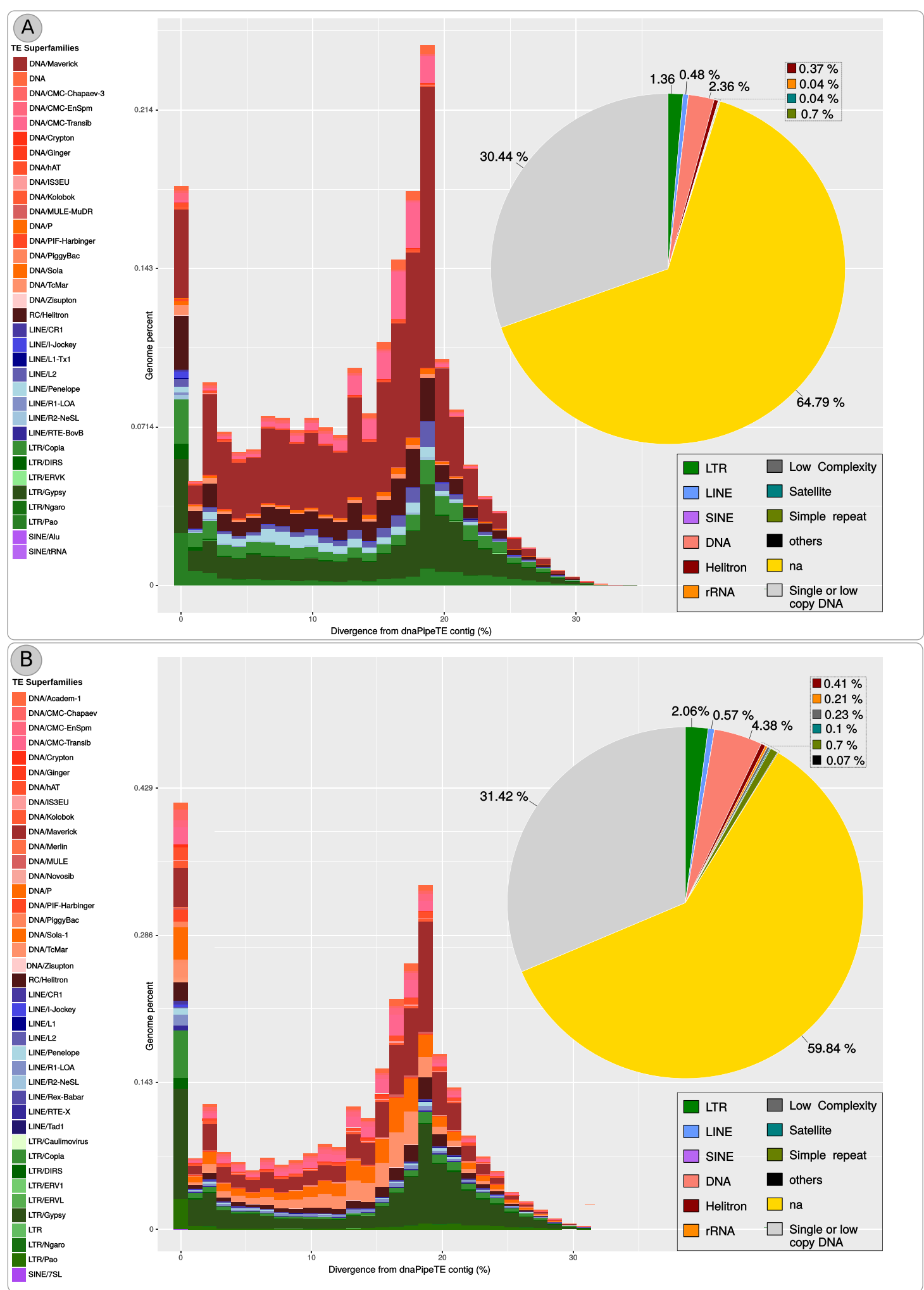

Fig. 1 Charts representing the proportion of each repetitive genomic component of the two wasp species. a braconid wasp and $\mathbf{b} L$. boulardi. Pie Chart with the overall proportion of reads by each repetitive type and landscape with proportion ( $y$-axis) and relative age analysis ( $\mathrm{x}$-axis - K2P) by each TE superfamily. Right legends correspond to major repetitive types found depicted in the pie chart graph (na - non annotated) and left legends correspond to TE superfamilies depicted in the landscape graph 


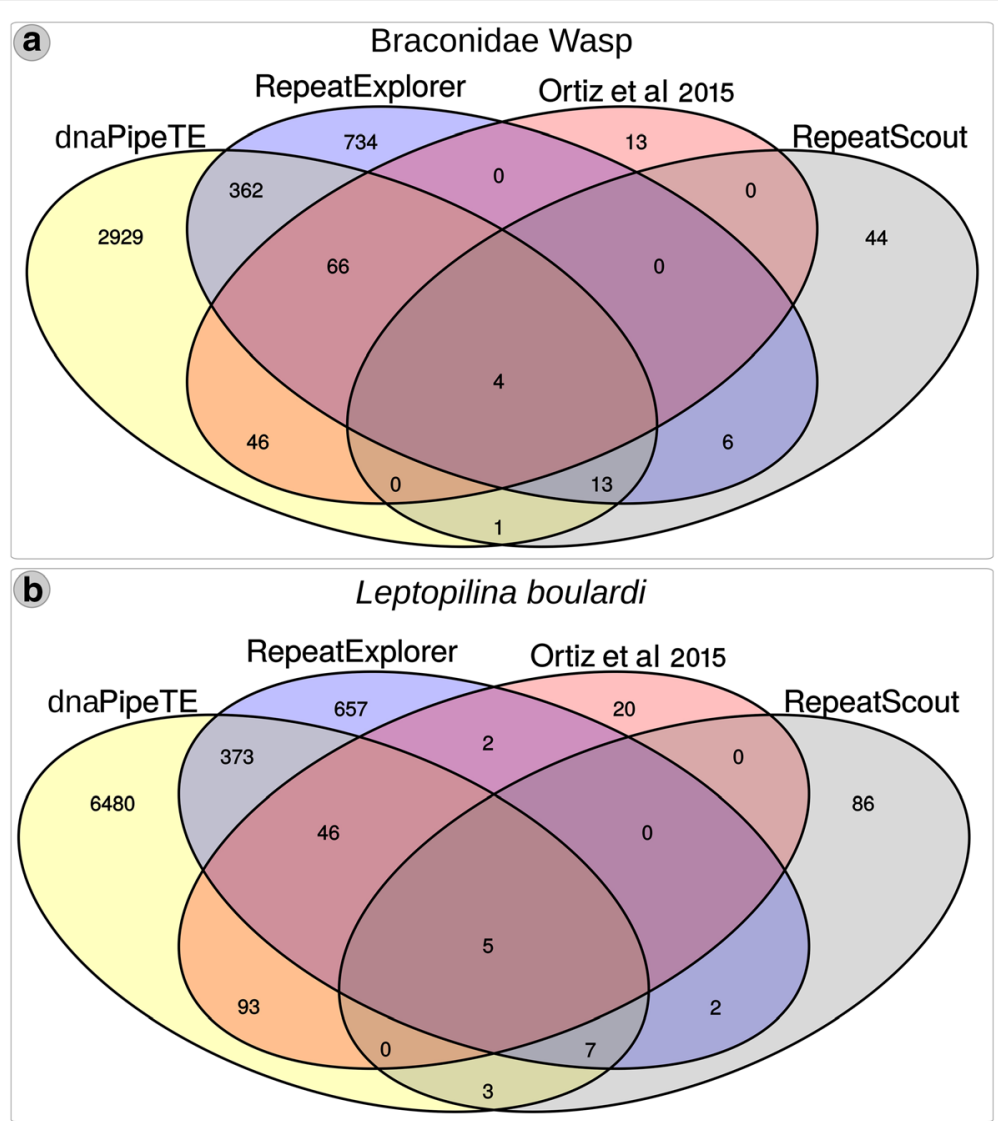

Fig. 2 Venn diagram with cd-hit-est clusterization of final TE contigs from each of the four analysis performed for TE characterization. a braconid wasp and $\mathbf{b}$ L. boulardi

(RepeatExplorer, dnaPipeTE, RepeatScout and the data provided by Ortiz et al. 2015) we clustered the resulting contigs following the $80-80$ rule [46]. We could detect that only a minor subset of 4 and 5 sequences in Braconidae and L. boulardi wasps were characterized by all 4 methodologies and a large overposition occurred between RE and dnaPipeTE with 362 and 373 sequences recovered by these two methods (Fig. 2a and b). The two methods that recovered the largest amount of unique elements was dnaPipeTE (2929 and 6480) and RepeatExplorer (734 and 657) for both wasps (Fig. 2a and b), but RepeatScout (44 and 86) and Ortiz et al. 2015 (13 and 20) library also presented unique sequences.

\section{Intragenomic dynamics}

Overall both wasps have a mobilome characterized by a mixture of ancient and young elements with representatives of all major superfamilies in the dnaPipeTE analysis (Fig. 1 $a$ and $b$ ). Additionally, we performed abundance and relative dating analysis within species on the RE reconstructed elements used in the phylogenetic analysis. A different relative abundance can be observed among elements reconstructed from the same genome. Such data can be seen in section B of Figs. 3, 4, 5 and 6. For instance, in clade II of Fig. 3b we can see that the element Helitron_CL245_Contig17_L_boulardi has the highest proportion of normalized reads in comparison to other closely related Helitron elements from $L$. boulardi genome. Another striking example can be seen in clade III in the Gypsy superfamily tree (Fig. 6b) where some elements presented a much higher relative abundance compared with other elements of the same species. Although we could see some patterns with a higher proportion of reads and lower K2P element age we could not detect any strong negative correlation (Pearson correlation between 0.3 and -0.3 ) inside each superfamily (data not shown). Overall, RE and dnaPipeTE relative age estimate were congruent showing that there is a young fraction of the mobilome in both wasps which is related to recent activity of these elements.

\section{Deeper TE superfamily characterization on RE reconstructed consensus}

Only contigs from RE analysis showing superfamily-specific proteins domains (Additional file 4) were used for phylogenetic reconstruction since they presented largest contig sizes 


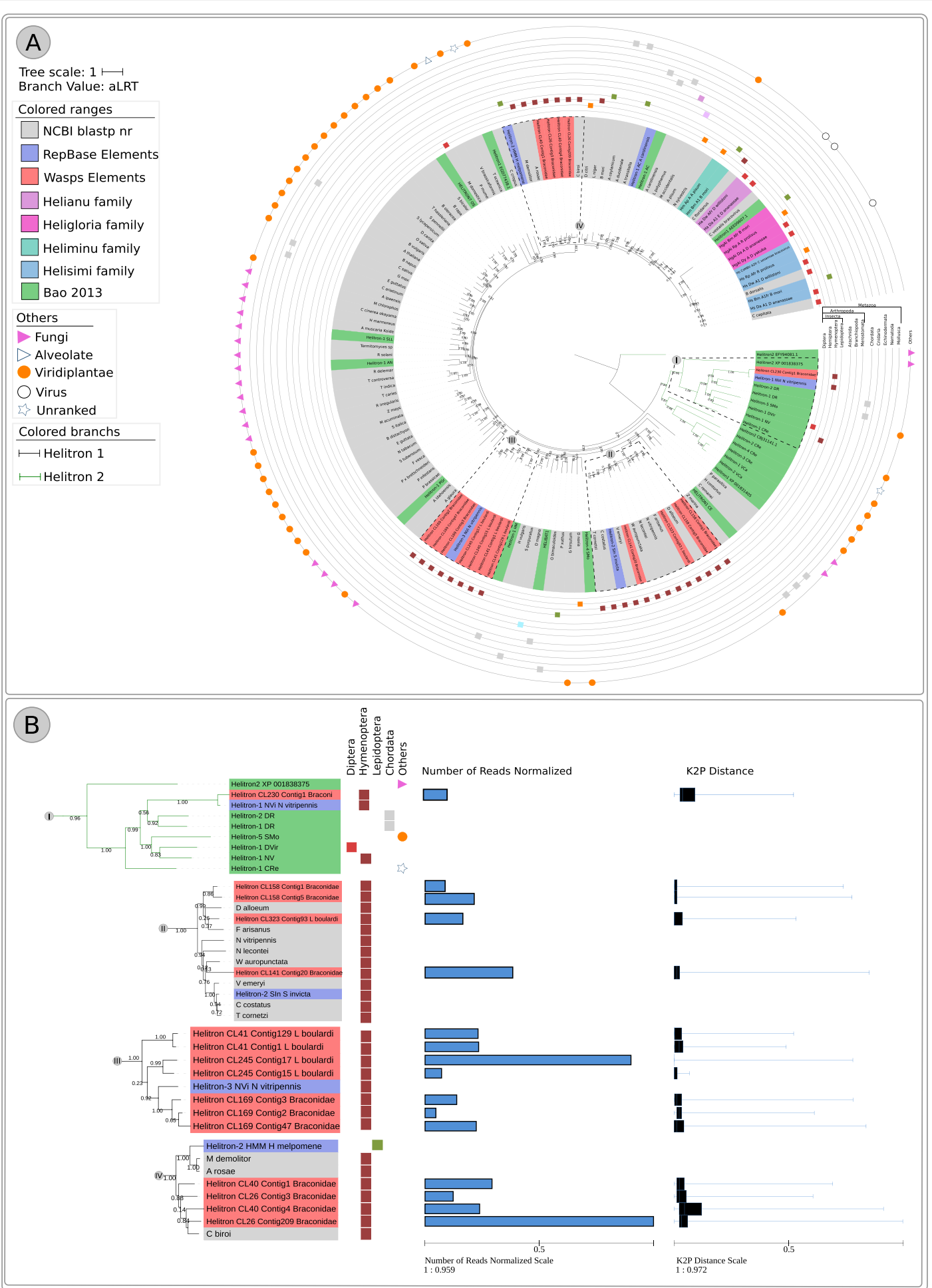

Fig. 3 Maximum likelihood Helitron phylogenetic reconstruction using Helicase protein sequences from Repbase, literature and NCBI search along with Helitron sequences from the wasps studied. Tip colors represent sequences recovered from RepBase - blue and NCBI database - grey. Moreover, orange, light green, purple and pink are the Helitron families described by Thomas et al. 2010 and black and green branch colors denotes Helitron 1 and 2 described by Bao and Jurka 2013 respectively. The number over nodes are aLRT estimates of node support. a Full phylogeny including all sequences sampled. Dashed squares represent the clades zoomed in part B. b Zoom on four clades encompassing wasp Helitrons. Blue bars are total reads used to assemble each contig normalized by the contig size and bluish boxplot is the average, and Q1/Q3 quartile and bars represent the maximum and minimum values. Online data about Helitron phylogeny is available on: https://itol.embl.de/tree/20013326113391523965160\# 


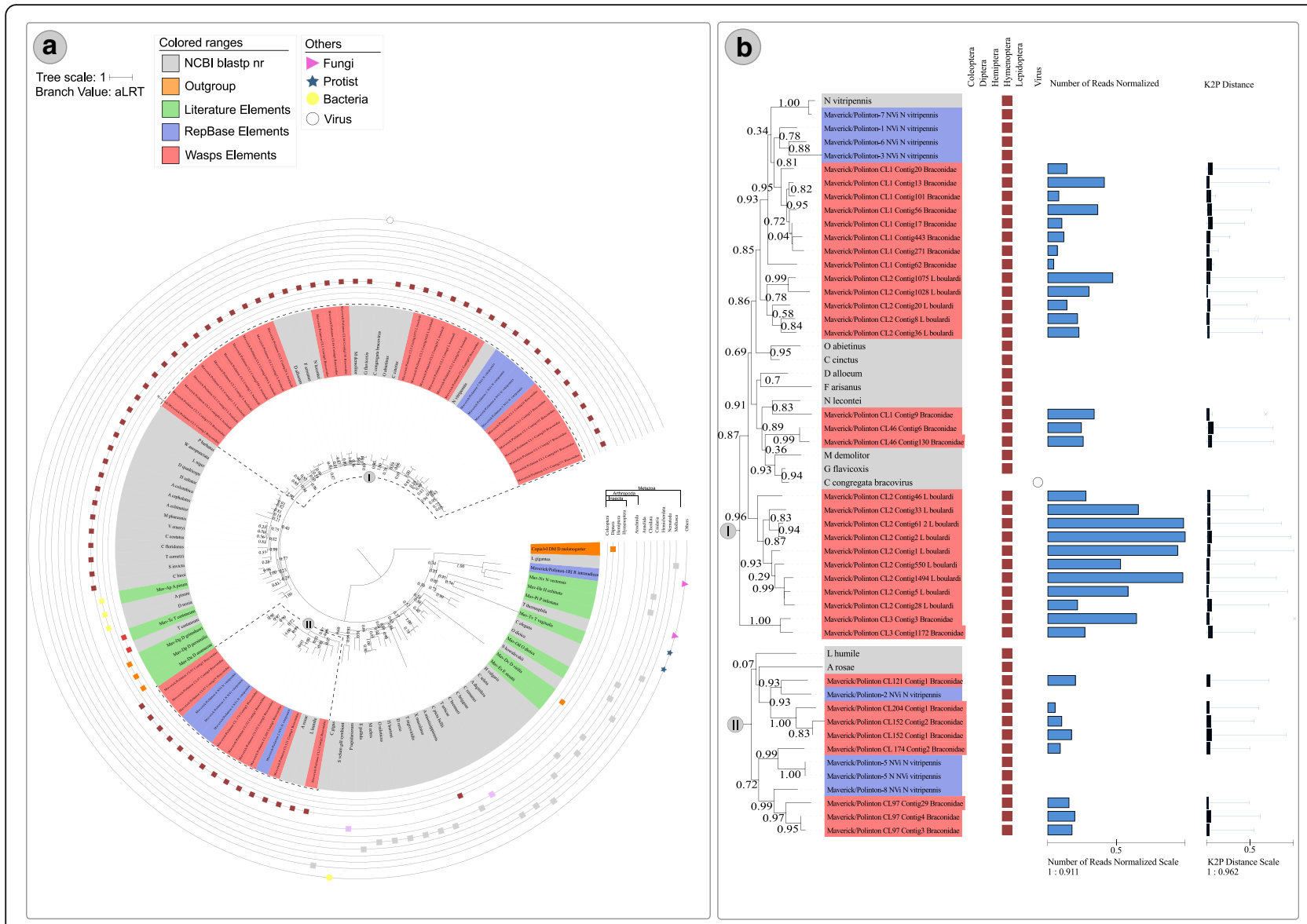

Fig. 4 Maximum likelihood Maverick/Polintons superfamily phylogenetic reconstruction using integrase protein sequences from Repbase, literature and NCBI search along with Maverick/Polintons sequences from the wasps studied. Tip colors represent sequences recovered from RepBase - blue, from the literature - green and NCBI database - black. The number over the nodes are aLRT node support. a Full phylogenetic tree and $\mathbf{b}$ Zoomed tree clades highlighted in the dashed squares from part A. Blue bars are total reads used to assemble each contig normalized by the contig size and bluish boxplot is the average and Q1/Q3 quartile and bars represent the maximum and minimum values. Online data about Maverick/Polintons phylogeny is available at: https:/itol.embl.de/tree/2001332610271781508755345\#

compared with the other strategies used in this study (Additional file 2). After manual alignment quality check, final alignments have smaller protein amino acid sequences ranging from around $62 \%$ of its original size (in Gypsy superfamily) to only $20 \%$ (in $L 2$ superfamily) (Additional file 5). Such large reduction in the Gypsy superfamily is probably due to the large diversity of proteins included in our analysis since this superfamily has the highest number of sequences used in the alignment and phylogenetic reconstruction (196) compared with other superfamilies. We were able to reconstruct the evolutionary history of seven superfamilies including elements from both $L$. boulardi and the braconid wasp (subsections below): Class II - DNA transposon - Helitron, Maverick/Polintons and Class I Retrotransposons - Copia, Gypsy, L2, BEL and Penelope (Additional file 6). Moreover, four phylogenetic trees were reconstructed only with $L$. boulardi sequences: Class II - DNA transposons - Chapaev and
Class I - Retrotransposons - Loa, I and R1 (Additional file 6). All sequences used in each phylogenetic analysis are presented in Additional file 7.

\section{The Helitron superfamily}

Known as rolling-circle elements, these TEs were first described in the genomes of plants (Arabidopsis thaliana and Oryza sativa) and in the nematode Caenorhabditis elegans [47]. Further studies identified homologous elements in several other genomes, such as protists, arthropods and mammals [48]. Helitrons are characterized by a RepHel region (Rep from Replication initiator and Hel from Helicase domain) and other protein domains such as Cysteine-Protease, Apurinic-Endonuclase, Zinc-Finger and Protein Replication A [48].

We used 16 contigs characterized with RE (11 from braconid wasp and 5 from $L$. boulardi) to reconstruct 


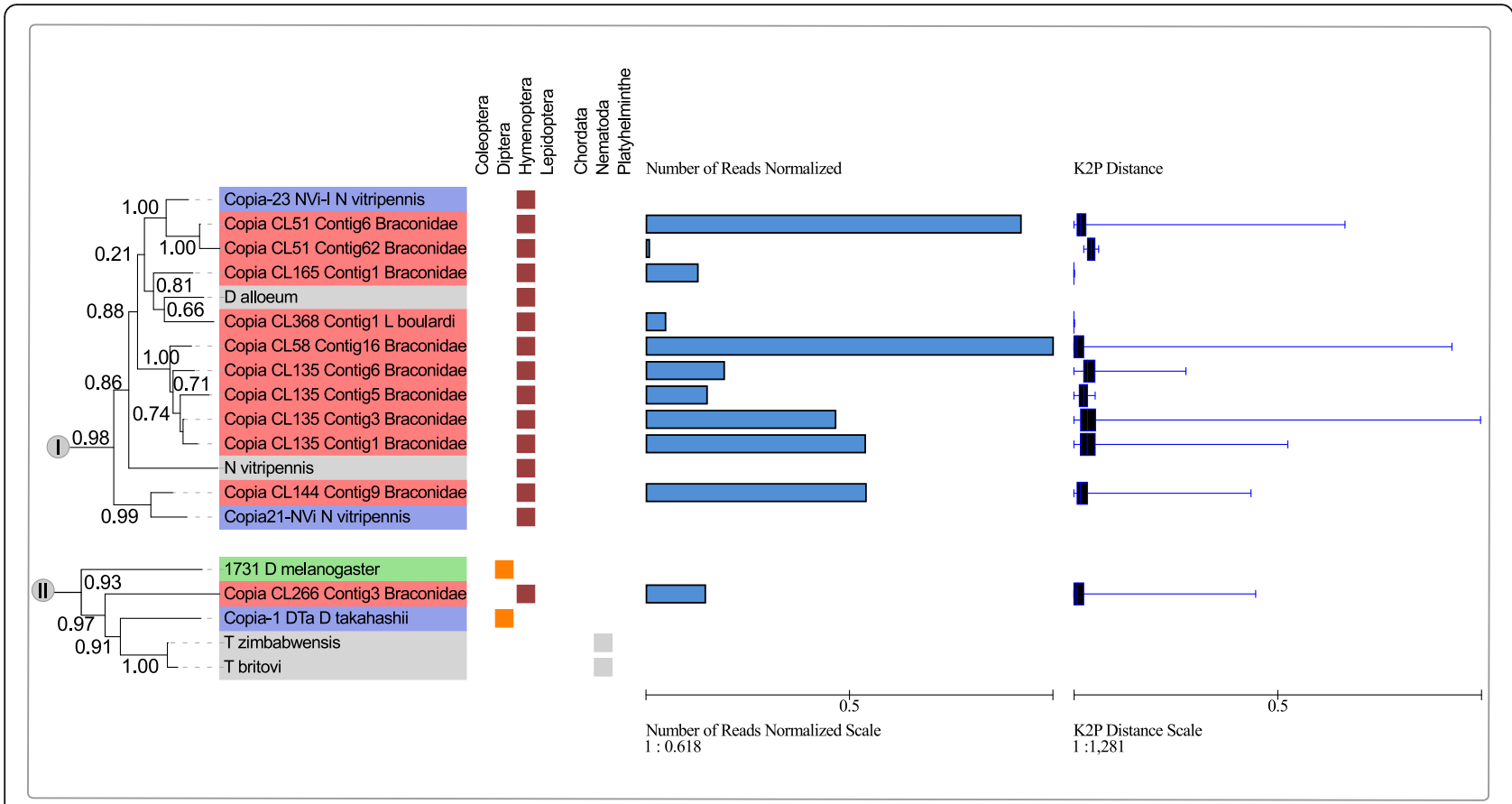

Fig. 5 Maximum likelihood of Copia superfamily phylogenetic reconstruction using full protein sequences from Repbase, literature and NCBI search along with Copia sequences from the wasps studied. Tip colors represent sequences recovered from RepBase - blue, from the literature - green and NCBI database - black. The number over the nodes are node support aLRT estimates. I and II are zoomed clades from full phylogeny which can be found in Additional file 9. Online data about Copia phylogeny is available at: https://itol.embl.de/tree/2001332610130171508862213

their evolutionary history. Those elements clustered into four divergent lineages (Fig. 3), two of those composed of sequences of TEs from both waps (Fig. 3 BII-BIII) and the other two with braconid sequence only (Fig. BI-BIV). All 16 contigs clustered outside of previous defined Helitron families [49] (orange, green, pink and purple in Fig. 3a) but 15 of those clustered inside of Helitron 1 while only 1 braconid sequences clustered inside Helitron 2 clade defined by Bao and Jurka 2013 (Fig. 3a black and green branches denotes Helitron 1 and 2 respectively): BI presents the single braconid Helitron clustered within the Helitron 2 cluster showing close phylogenetic relationship with the Helitron-1 element from N. vitripennis available at Repbase (Fig. 3b). BII clade grouped Helitrons from L. boulardi and braconid wasps with other wasp species such as $N$. vitripennis, Diachasma alloeum (Braconidae), Fopius arisanus (Braconidae) and four ant species (Wasmannia auropunctata, Vollenhovia emeryi, Cyphomyrmex costatus, Trachymyrmex cornetzi) (Fig. 3b). BIII clade grouped three braconid and four L. boulardi elements with Helitron-3 element from $N$. vitripennis described in Repbase (Fig. 3b). BIV grouped Braconidae Helitrons sequences with an ant, Cerapachys biroi Helitron sequence found in NCBI (Fig. 3 a and b, Additional file 8). Overall, Helitrons from wasp species clustered with each other and were closely related to ants, being congruent with host species phylogeny [50].

\section{The Maverick/Polintons superfamily}

TEs of this superfamily have been found in protist, fungi and animal genomes and are characterized as one of the largest and structurally complex TEs described to date (10-15 kb) which codify four proteins probably involved in their transposition mechanism: DNA-Polymerase-B, Retroviral-Integrase, Cysteine-Protease and ATPase [51, 52].

Here we identified $36 \mathrm{RE}$ contigs with Retroviral-Integrase protein domains (22 from braconid wasp and 14 from $L$. boulardi) which were used to reconstruct their evolutionary history (Fig. 4). Wasps Maverick/Polintons sequences clustered into two major clades (I and II in Fig. $4 \mathrm{a}$ and b). Clade I presented several contigs of both wasps and several other wasp sequences from databases. Highly supported subclades show a basal clade with only two braconid sequences, a second clade with only $L$. boulardi Maverick/Polintons sequences, a third clade with a single braconid wasp element clustered with Neodiprion lecontei sequence and a clade with two braconid wasp sequences, a fourth clade with five L. boulardi elements and eight braconid wasp elements clustering with $N$. vitripennis elements (Fig. 4b). While clade II has two clear subclades with braconid wasp Maverick/Polintons and N. vitripennis, Linepithema humile and Athalia rosae sequences (Fig. 4b). Overall, Maverick/Polintons evolution followed vertical transmission, clustering wasp sequences characterized here with other wasp and Formicidae sequences available. 


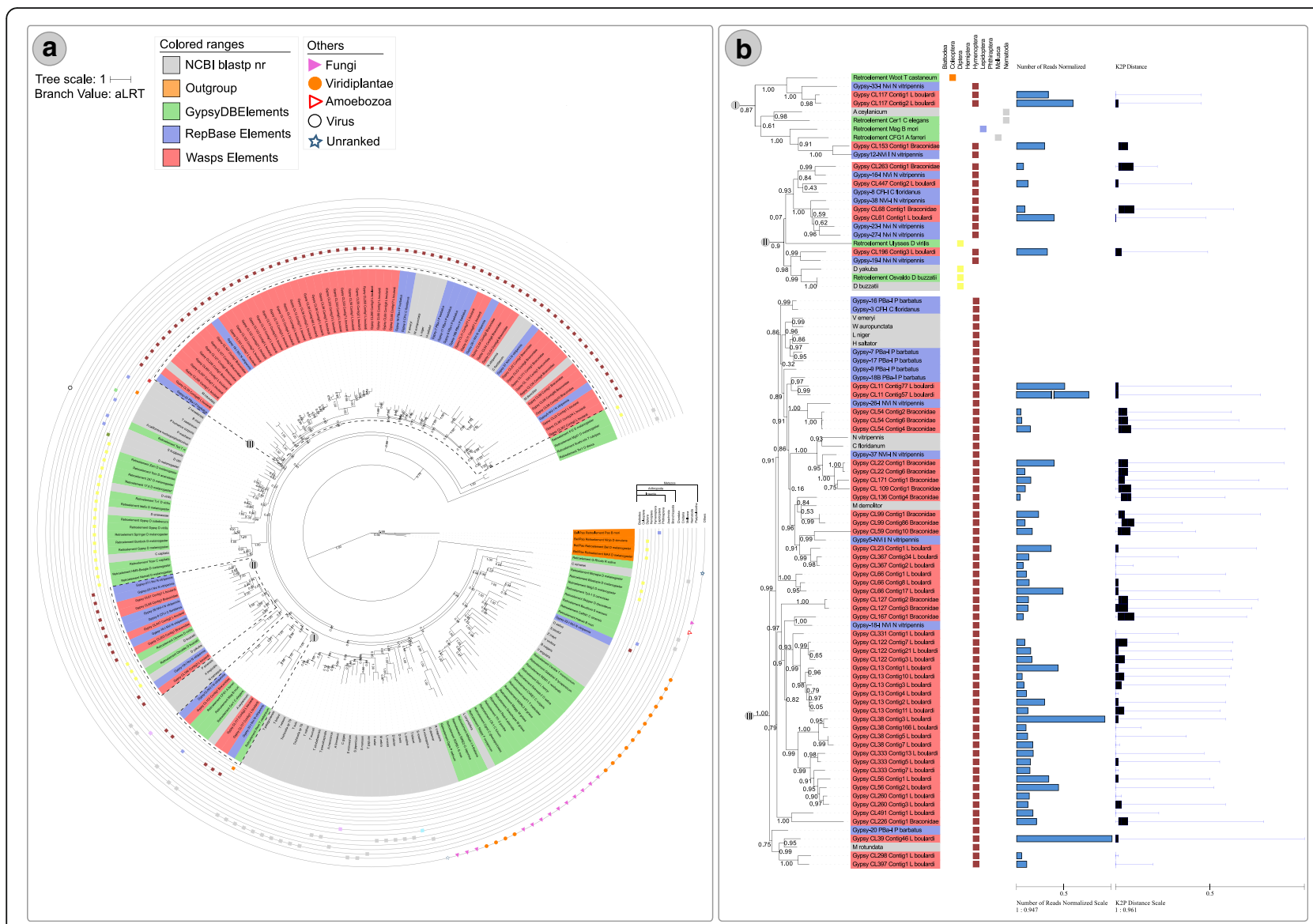

Fig. 6 Maximum likelihood of Gypsy superfamily phylogenetic reconstruction using full protein sequences from Repbase, literature and NCBI search along with Gypsy sequences from the wasps studied. Tip colors represent sequences recovered from RepBase - blue, from the literature - green and NCBI database - black. The number over nodes are aLRT estimates of node support. a Full phylogenetic tree and $\mathbf{b}$ Zoomed tree clades highlighted in the dashed square from part A. Blue bars are total reads used to assemble each contig normalized by the contig size and bluish boxplot is the average, and Q1/Q3 quartile and bars represent the maximum and minimum values. Online data about Gypsy phylogeny is available at: https://itol.embl.de/tree/177183205251262901509016275

\section{The Copia superfamily}

TEs of this superfamily were first identified in the Drosophila melanogaster genome [53] and subsequently in plant genomes [54], but several studies showed that these elements are widely distributed among eukaryotic taxa. Elements from this superfamily codify a single ORF with two domains (GAG and POL - GAG is associated with structural proteins responsible for TE genetic material packing in viral like particles (VLPs - similar to retroviruses), POL genes which are responsible for TE replication and translocation [55]) and long terminal repeats in both $5^{\prime}$ and $3^{\prime}$ element extremities [56].

Eleven RE contigs presenting a reverse-transcriptase domain were analyzed phylogenetically (ten from braconid wasp and 1 from $L$. boulardi) (full phylogeny can be found in Additional file 9 and clades bearing TEs from wasps in Fig. 5). Copia elements clustered into two divergent lineages, one encompassing 9 braconid wasp elements and a single $L$. boulardi element (Fig. 5 - Clade I). Clade I elements clustered with three $N$. vitripennis (Pteromalidae) elements (Copia-23 and Copia-21) and one element from the parasitoid wasp D. alloeum (Braconidae) (Fig. 5 - Clade I) showing an early branch of this clade composed of a Copia element from Trichogramma pretiosum, another parasitoid wasp, but from Trichogrammatidae family. Clade II clustered a single braconid wasp element with two Drosophila and two nematodes species from the Trichinella genus with a high node support (0.91-1, Fig. 5). Such elements presented a distance ranging from 1.22 to 1.59 amino acid changes per site (Additional file 10- red shaded cells).

\section{The Gypsy superfamily}

TEs of this superfamily are abundant in plant and animal genomes [57]. They are characterized by two ORFs, 
GAG and POL, similar to the Copia superfamily, but with different protein domains positions [46].

We identified a total of fifty-six RE contigs with the reverse-transcriptase domain, 18 from the braconid wasp and 38 from L. boulardi. The Gypsy superfamily is the most diverse superfamily found among those parasitic wasps (Fig. 6). Three large clades encompassing wasp Gypsy elements can be seen (Fig. 6a and b - Clade I, II and III). Overall, Gypsy elements from braconid wasp formed clusters with $N$. vitripennis and $L$. boulardi elements (Fig. 5a and b - Clade I and II). However, some clades were found composed by ant and bees sequences closely related to wasps Gypsy elements (Fig. 6b). For instance, Gypsy_CL11_Contig77_L_boulardi and Gypsy_CL11_Contig57_L_boulardi elements clustered with Gypsy-18 B_PBa-I_P_barbatus, an ant species Pogonomyrmex barbatus with high branch support (0.97) (Fig. 6b - Clade III), and presented an amino acid distance of 0.75 and 0.76 amino acid changes per site. Another example in the same clade is Gypsy_CL39_Contig1_L_boulardi which grouped with a bee, Megachile rotundata, an element with high branch support (0.96) and an amino acid distance of 0.49 amino acid substitution per site (Fig. 6b - Clade III).

Other interesting branching pattern emerging from the Gypsy tree were two clearly defined clusters with only $L$. boulardi elements which suggests that those elements were amplified and diversified successfully in this genome (Fig. 5b - Clade I).

\section{Discussion}

The extraordinary rate in which new genomes are being sequenced allows researchers to have a better view of genome evolution in several different taxa and test the consistency of major patterns driving genome expansion and diversification. However, the mobilome, a dynamic and large fraction of many genomes, is disregarded mainly due to its inherent complexity associated with a long-lasting view that it is not important for understanding the genome evolutionary dynamics. Although some efforts have been made to better characterize the mobilome, we only have a precise characterization for model organisms. Here we described the mobilome of two non-model organisms, a Braconidae wasp (probably a new species from the Aphidius genus) and L. boulardi wasp species showing that they have a diverse mobilome and a mixture of ancient and young elements. Moreover, TE content and superfamily diversity of DNA transposons differs substantially between them and with other previous studied wasp species.

TE content in eukaryotic genomes varies greatly with some species apparently free of such parasites up to genomes composed of 60 to $80 \%$ [2]. In insect the TE content ranges from $<1 \%$ in Belgica antarctica to $60 \%$ in Locusta migratoria and Aedes albopictus genomes [21]. Species from the Hymenoptera order also have a large variation in TE content as the bee Apis mellifera 7.57\% [58], and the ant species Cardiocondyla obscurior 7.18\% [59], and the Camponotus floridanus 15.62\% [60], Harpegnathos saltator $27.53 \%$ ant species [60]. Wasps mobilome characterization only exists for three Nasonia genomes having a TE content of around $25.8 \%$ of their genomes [24] although a recent publication reported a lower estimate: 20\% [59]. Braconidae and $L$. boulardi wasps studied here showed the smallest TE contents (considering both RE and dnaPipeTE results braconid - 5.86/4.57 and L. boulardi - 5.22/7.42) compared other Hymenopteran and Nasonia genomes. However, we need to keep in mind that mobilome comparison among studies is a hard task mainly due to different genome quality and approaches used for TE detection and annotation (see discussion below and references - $[23,61]$ ) and that the genomes studied here represent only a tiny subset of wasp species diversity.

Known insect genomes usually have a higher proportion of Class I than Class II TEs [21]. However, reported Hymenopteran genomes have a higher proportion of Class II (six ants genomes) than Class I (2 genomes including $N$. vitripennis), while Apis mellifera showed an almost equal amount of these classes [58, 59]. Braconidae and L. boulardi wasps presented a higher proportion of Class II than Class I TEs (Fig. 1). Such differences can be partially explained by the large amount of Maverick/Polintons superfamily in both wasp species, being the most abundant superfamily of braconid wasp genome in both RE and dnaPipeTE analysis, and the third and second more abundant in $L$. boulardi genome in $\mathrm{RE}$ and dnaPipeTE results (Additional file 3). Other Hymenopterans genomes, such as Atta cephalotes, $H$. saltator and Acromyrmex echinatior ant species, have a low Maverick/Polintons genomic proportion ranging from $0.509,0.543$ and $0.408 \%$ compared with the total Class II TE content which was estimated around 7, 7.5 and $8 \%$ respectively. While $N$. vitripennis presented a Class II TE content of $7.8 \%$ and a Maverick/Polintons content of $1.452 \%$ [59]. Taking the data presented above, we estimate that the contribution of Maverick/Polintons to the total Class II TEs content presented higher values in N. vitripennis and Braconidae wasps studied here (18.61 and 32.01\%), two species from sister families Braconidae and Pteromalidae, than in the three ant species and $L$. boulardi species where such contribution varied between 5.1 in $A$. echinator up to $12.40 \%$ in L. boulardi (7.24\% in H. saltator and $7.27 \%$ in A. cephalotes). This data suggests that Maverick/Polintons superfamily is an important component of the Pteromalidae and Braconidae wasps genomes that probably expanded in the ancestral of these two families around 213 MYA (http://www.timetree.org/). Maverick/Polintons seems to contribute to Class II TE in L. boulardi as well, besides a high abundance of Sola, Tc1-mariner, Helitron and Transib superfamilies is also observed (Additional file 3). 
The mobilome of those two wasp species was characterized before using a homology-based approach obtaining a total of 20 superfamilies [29]. In this study, we used a combination of two de novo approaches based on $\mathrm{TE}$ reconstruction from raw reads (RE and dnaPipeTE) and one de novo approach that characterize TEs from assembled genomes (RS) in combination with the original TEs described by Ortiz et al. 2015 to generate a final TE dataset for the studies wasps. We were able to identify several new TEs superfamilies and a large overpositon of TEs recovered could be observed among the methodologies employed (Fig. 2a and b). However, each strategy recovered TE contigs with specific features. Given the non-model species studied here and the increase in TE superfamilies and TE consensus size detected by the de novo approach directly from raw reads, such strategy seems to be more appropriate to characterize mobilomes of non-model organisms. Anyhow, those approaches should be used complementary to each other in order to have a more complete view of the mobilome.

The identification of a large number of contigs allowed the extraction of potential coding regions and identification of several TE specific domains which allowed us to get further insight into their evolution (Additional file 4). We were able to reconstruct seven superfamily-level phylogenetic trees, representing the most abundant and diverse superfamilies found by RE. In general, TE superfamilies from braconid wasp studied here are more diversified, that is, have a larger number of contigs (which represent TE families) than $L$. boulardi TE superfamilies with the exception of the Gypsy superfamily. Clustering patterns showed that elements from both wasps usually grouped closely with elements characterized in N. vitripennis, other wasps, or ant and bee species, all from the Hymenoptera order. Moreover, braconid wasp elements are more related to $N$. vitripennis elements than to L. boulardi elements which reflects the phylogenetic relationship of the host species: Braconidae and Pteromalidae (N. vitripennis) wasp families are more phylogenetically related than Figitidae family (L. boulardi) [29] which is in agreement with the results presented before regarding the diversity and abundance of Maverick/Polinton on $N$. vitripennis and braconid wasp. Overall, branching patterns suggests that the majority of elements from the two wasps are evolving through vertical transmission. However, some phylogenetic clusters encompass closely related TEs from host species that split several million years ago can be seen in the Gypsy phylogenetic tree: I - L. boulardi sequences clustered with Megachile rotundata, a bee species, presenting around $70 \%$ of similarity at the protein level. These two species diverged from each other around 203 Mya (http://www.timetree.org/). Therefore, at least for Gypsy superfamily our data suggests one ancient horizontal transfer event between wasp and bees species although further evidences are needed in order to better evaluate such event.

Another interesting point is that even knowing that Drosophila genomes have one of the best-characterized mobilomes [62], we could not find any traces of potential horizontal transfer events between wasps and flies, which supports our previous findings that Drosophila parasitoid wasps, and Drosophila hosts do not exchange TEs through HT, and that VLPs injected by these wasps are not an important TE vector [29]. This is in contrast with several horizontal transfer events mediated by Lepidoptera parasitoid wasps and their VLPs [25-27, 63]. These contradictory findings suggest that each vector-parasite relationship has different characteristics that either allow or act as a barrier for HT between species and highlights the importance of taking into account non-model organisms when extrapolating results on the evolution of TEs.

Based on our broad analysis including several protein sequences from different databases, we were able to reconstruct comprehensive superfamily phylogenetic trees which brought new information about the evolution of several TEs superfamilies. Helitron superfamily tree obtained in this work is the first tree including such extensive Helitron diversity. Most Helitron studies focused in phylogenetic trees at the nucleotide level which hindered analysis of more distant homologous sequences as well as confident reconstruction of deep ancestral nodes with confidence $[49,64]$. There is only one study reporting deep branch nodes of Helitron superfamily but that was analyzed with only 29 protein sequences [65]. Our comprehensive tree recovered the two major monophyletic Helitrons clades described by Bao and Jurka 2015 showing that parasitoid wasps sequences mostly belong to the Helitron 1 clade and only one sequence characterized in this study clustered in the Helitron 2 clade. Regarding Maverick/Polintons, the last published and more comprehensive analysis used all sequences from this superfamily available at Repbase (56 elements) to reconstructs their evolutionary history [66]. Here we used 88 elements gathered from the literature [67] and available at NCBI along with 36 elements reconstructed from braconid and $L$. boulardi genomes. We obtained the same two major clades with similar topologies recovered by Haapa-Paananem et al. 2014, but our large sampling allowed us to show that ant Maverick/Polintons are the sister group of wasp Maverick/Polintons and deep understand the diversity of Maverick/Polintons from several species of wasps including the one focused on this study.

Regarding the abundance of elements and their intragenomic dynamics we could detect that some elements probably have a higher copy number which is related to 
a more successful amplification in the host genome. Moreover, we detected that the wasps mobilomes studied here have an ancient mobilome, which is in agreement with the vertical inheritance signal supported by the phylogenetic analysis, but with a similar amount of young TE families which is likely related with recent activity of these elements.

\section{Conclusion}

This study characterized two non-model wasp mobilomes shedding new light on the evolution of those elements and hosts. We detected several TE superfamilies not described before for those species, showed that Maverick/Polintons compose an abundant genomic component in Pteromalidae and Braconidae wasps, revisited some TE superfamily phylogeny showing that most of the wasps TEs are evolving vertically and evidenced that the two wasps mobilomes investigated here have a miscellaneous of ancient and young elements which likely contribute to the intragenomic dynamics of such understudied taxa $[68-70]$.

\section{Additional files}

Additional file 1: Workflow of softwares used in this study. A) pipelines and softwares used to complementary characterize the mobilome of both wasp species and derivate a final TE dataset. B) Evolutionary analysis performed only with RepeatExplorer contigs per superfamily. (PDF 31 kb)

Additional file 2: Features of TE contigs recovered by different approaches. (DOCX $8 \mathrm{~kb}$ )

Additional file 3: Summary of superfamilies abundance per wasp species analyzed. (DOCX $13 \mathrm{~kb}$ )

Additional file 4: Contigs description and TE conserved domains found. (XLSX $38 \mathrm{~kb}$ )

Additional file 5: Protein length before and after manual alignment edition. (XLSX $46 \mathrm{~kb}$ )

Additional file 6: Species-specific superfamily trees reconstructed by maximum likelihood. The number on branches denote the aLRT branch support. (PDF $4864 \mathrm{~kb}$ )

Additional file 7: Final TE contigs after redundancy removal with cd-hitest characterized in this study. (FASTA $7362 \mathrm{~kb}$ )

Additional file 8: Helitron phylogenetic tree with complete coding regions of RepBase elements. (PDF $59 \mathrm{~kb}$ )

Additional file 9: Copia superfamily tree reconstructed by maximum likelihood. Numbers on branches denote the aLRT branch support. (PDF $641 \mathrm{~kb})$

Additional file 10: Amino acid distance between TEs proteins used in the phylogenetic reconstruction. (XLSX $914 \mathrm{~kb})$

\section{Abbreviation}

NCBI: National Center for Biotechnology Information; STDEV: standard deviation; TE: transposable element; VLP: viral-like particle

\section{Acknowledgments}

We thanks very much Antonio Mauro Rezende for his helpful and essential assistance with pipelines implementation.

\section{Authors' contributions}

AFS and FZD analyzed and interpreted the data. AFS, FZD, GLW and ELSL wrote the manuscript. All authors read and approved the final manuscript.
Ethics approval and consent to participate

Not applicable.

\section{Consent for publication}

Not applicable.

Competing interests

The authors declare that they have no competing interests.

\section{Publisher's Note}

Springer Nature remains neutral with regard to jurisdictional claims in published maps and institutional affiliations.

\section{Author details}

${ }^{1}$ Pós Graduação em Biociências e Biotecnologia em Saúde, Instituto Aggeu Magalhães (IAM), Recife, Pernambuco, Brazil. ${ }^{2}$ Departamento de Bioquímica e Biologia Molecular, Universidade Federal de Santa Maria, Santa Maria, Rio Grande do Sul, Brazil. ${ }^{3}$ Departamento de Entomologia, Instituto Aggeu Magalhães (IAM), Fundação Oswaldo Cruz (FIOCRUZ/PE), Recife, Pernambuco, Brazil.

Received: 11 January 2018 Accepted: 19 June 2018

Published online: 07 July 2018

\section{References}

1. McClintock B. The Fusion of Broken Ends of Chromosomes Following Nuclear Fusion. Proc. Natl. Acad. Sci. United States Am. 1942;28:458-63.

2. Pritham EJ. Transposable elements and factors influencing their success in eukaryotes. J. Hered. 2009;100:648-55.

3. Piskurek O, Jackson DJ. Transposable elements: from DNA parasites to architects of metazoan evolution. Genes. 2012;3:409-22.

4. Charlesworth B, Langley CH. The evolution of self-regulated transposition of transposable elements. Genetics. 1986;112:359-83.

5. Kapitonov W, Jurka J. RAG1 core and V(D)J recombination signal sequences were derived from Transib transposons. PLoS Biol. 2005;3:e181.

6. Biessmann H, Valgeirsdottir K, Lofsky A, Chin C, Ginther B, Levis RW, et al. HeT-A, a transposable element specifically involved in "healing" broken chromosome ends in Drosophila melanogaster. Mol. Cell. Biol. 1992;12:3910-8

7. Miller WJ, McDonald JF, Pinsker W. Molecular domestication of mobile elements. Genetica. 1996;100:261-70.

8. Kidwell MG. Transposable elements and the evolution of genome size in eukaryotes. Genetica. 2002;115:49-63.

9. Lerat E. Identifying repeats and transposable elements in sequenced genomes: how to find your way through the dense forest of programs. Heredity. 2010;104:520-33.

10. Goubert C, Modolo L, Vieira C, ValienteMoro C, Mavingui P, Boulesteix M. De novo assembly and annotation of the Asian tiger mosquito (Aedes albopictus) repeatome with dnaPipeTE from raw genomic reads and comparative analysis with the yellow fever mosquito (Aedes aegypti). Genome Biol. Evol. 2015:7:1192-205.

11. Zytnicki M, Akhunov E, Quesneville H. Tedna: a transposable element de novo assembler. Bioinformatics. 2014;30:2656-8.

12. Rius N, Guillén Y, Delprat A, Kapusta A, Feschotte C, Ruiz A. Exploration of the Drosophila buzzatii transposable element content suggests underestimation of repeats in Drosophila genomes. BMC Genomic. 2016;17:344.

13. Li R, Ye J, Li S, Wang J, Han Y, Ye C, et al. ReAS: Recovery of Ancestral Sequences for Transposable Elements from the Unassembled Reads of a Whole Genome Shotgun. PLoS Comput. Biol. 2004;1:313-21.

14. Novák P, Neumann P, Macas J. Graph-based clustering and characterization of repetitive sequences in next-generation sequencing data. BMC Bioinforma. 2010;11:378.

15. Novák P, Neumann P, Pech J, Steinhaisl J, Macas J. RepeatExplorer: a Galaxybased web server for genome-wide characterization of eukaryotic repetitive elements from next-generation sequence reads. Bioinforma. 2013;29:792-3.

16. Shah AB, Schielzeth H, Albersmeier A, Kalinowski J, Hoffman Jl. Highthroughput sequencing and graph-based cluster analysis facilitate microsatellite development from a highly complex genome. Ecol. Evol. 2016;6:5718-27. 
17. García G, Ríos N, Gutiérrez V. Next-generation sequencing detects repetitive elements expansion in giant genomes of annual killifish genus Austrolebias (Cyprinodontiformes, Rivulidae). Genetica. 2015;143:353-60.

18. Goubert C, Henri H, Minard G, Valiente Moro C, Mavingui P, Vieira C, et al. High-throughput sequencing of transposable element insertions suggests adaptive evolution of the invasive Asian tiger mosquito towards temperate environments. Mol. Ecol. 2017;26:3968-81.

19. Novák P, Hřibová E, Neumann P, Kobližková A, Doležel J, Macas J. Genomewide analysis of repeat diversity across the family Musaceae. PloS one. 2014; 9:e98918.

20. Sessegolo C, Burlet N, Haudry A. Strong phylogenetic inertia on genome size and transposable element content among 26 species of flies. Biol. Lett. 2016;12:20160407.

21. Maumus F, Fiston-Lavier A-S, Quesneville H. Impact of transposable elements on insect genomes and biology. Curr. Opin. Insect Sci. 2015;7:30-36.

22. Muñoz-Diez C, Vitte C, Ross-lbarra J, Gaut BS. Tenaillon MI. Using Nextgen Sequencing to Investigate Genome Size Variation and Transposable Element Content. In: Grandbastien MA., Casacuberta J. (eds) Plant Transposable Elements. Topics in Current Genetics, Springer, Berlin, Heidelberg. 2012;24:41-58.

23. Elliott TA, Gregory TR. Do larger genomes contain more diverse transposable elements? BMC Evol. Biol. 2015;15:69.

24. Werren JH, Richards S, Desjardins CA, Niehuis O, Gadau J, Colbourne JK, et al. Functional and evolutionary insights from the genomes of three parasitoid Nasonia species. Sci. 2010;327:343-8.

25. Herniou EA, Huguet E, Thézé J, Bézier A, Periquet G, Drezen J-M. When parasitic wasps hijacked viruses: genomic and functional evolution of polydnaviruses. Philos. Trans. R. Soc. London. Ser. B, Biol. Sci. 2013;368: 20130051.

26. Gilbert C, Cordaux R. Viruses as vectors of horizontal transfer of genetic material in eukaryotes. Curr. Opin. Virol. 2017;25:16-22.

27. Gilbert C, Peccoud J, Chateigner A, Moumen B, Cordaux R, Herniou EA. Continuous Influx of Genetic Material from Host to Virus Populations. PLoS Genet. 2016;12:e1005838.

28. Geib SM, Liang GH, Murphy TD, Sim SB. Whole Genome Sequencing of the Braconid Parasitoid Wasp, an Important Biocontrol Agent of Pest Tepritid Fruit Flies. G3. 2017;7:2407-11.

29. Ortiz MF, Wallau GL, Graichen DÂS, Loreto ELS. An evaluation of the ecological relationship between Drosophila species and their parasitoid wasps as an opportunity for horizontal transposon transfer. Mol. Genet. Genomic. 2015;290:67-78.

30. Oliveira DS, Gomes TMFF, Loreto ELS. The rearranged mitochondrial genome of Leptopilina boulardi (Hymenoptera: Figitidae), a parasitoid wasp of Drosophila. Genet. Mol. Biol. 2016;39:611-5.

31. Robe LJ, De Ré FC, Ludwig A, Loreto ELS. The Drosophila flavopilosa species group (Diptera, Drosophilidae): An array of exciting questions. Fly. 2013;7:59-69.

32. Bao W, Kojima KK, Kohany O. Repbase Update, a database of repetitive elements in eukaryotic genomes. Mob. DNA. 2015;6:11.

33. Huang X, Madan A. CAP3: A DNA sequence assembly program. Genome Res. 1998:9:868-77

34. Pagán HJT, Macas J, Novák P, McCulloch ES, Stevens RD, Ray DA. Survey sequencing reveals elevated DNA transposon activity, novel elements, and variation in repetitive landscapes among vesper bats. Genome Biol. Evol. 2012;4:575-85

35. Price $A L$, Jones NC, Pevzner PA. De novo identification of repeat families in large genomes. Bioinformatics. 2005;21:i351-8.

36. Li W, Jaroszewski L, Godzik A. Clustering of highly homologous sequences to reduce the size of large protein databases. Bioinformatics. 2001;17:282-3.

37. Rice P, Longden I, Bleasby A. EMBOSS: the European Molecular Biology Open Software Suite. Trends Genet. 2000;16:276-7.

38. Marchler-Bauer A, Bryant SH. CD-Search: protein domain annotations on the fly. Nucleic acids Res. 2004;32:W327-31.

39. Jurka J, Klonowski P, Dagman V, Pelton P. CENSOR-a program for identification and elimination of repetitive elements from DNA sequences. Comput Chem. 1996;20:119-21.

40. Rombel IT, Sykes KF, Rayner S, Johnston SA. ORF-FINDER: a vector for highthroughput gene identification. Gene. 2002;282:33-41.

41. Katoh K, Misawa K, Kuma K, Miyata T. MAFFT: a novel method for rapid multiple sequence alignment based on fast Fourier transform. Nucleic Acids Res. 2001;30:3059-66.
42. Guindon S, Delsuc F, Dufayard J-F, Gascuel O. Estimating maximum likelihood phylogenies with PhyML. Methods Mol. Biol. 2008;537:113-37.

43. Cummings MP. FigTree. Dict. Bioinforma. Comput. Biol: Wiley; 2004.

44. Letunic I, Bork P. Interactive tree of life (iTOL) v3: an online tool for the display and annotation of phylogenetic and other trees. Nucleic acids Res. 2016:44:W242-5.

45. Kumar S, Stecher G, Tamura K. MEGA7: Molecular Evolutionary Genetics Analysis Version 7.0 for Bigger Datasets. Mol. Biol. Evol. 2016;33:1870-4.

46. Wicker T, Sabot F, Hua-Van A, Bennetzen JL, Capy P, Chalhoub B, et al. A unified classification system for eukaryotic transposable elements. Nat. Rev. Genet. 2006:8:973-82.

47. Kapitonov W, Jurka J. Rolling-circle transposons in eukaryotes. Proc. Natl. Acad. Sci. United States Am. 2000;98:8714-9.

48. Kapitonov W, Jurka J. Helitrons on a roll: eukaryotic rolling-circle transposons. Trends Genet. 2007;23:521-9.

49. Thomas J, Schaack S, Pritham EJ. Pervasive horizontal transfer of rollingcircle transposons among animals. Genome Biol. Evol. 2010;2:656-64.

50. Maddison DR, Schulz K-S, Maddison WP. The Tree of Life Web Project. Zootaxa. 2006:19-40.

51. Kapitonov W, Jurka J. Self-synthesizing DNA transposons in eukaryotes. Proc. Natl. Acad. Sci. United States Am. 2005;103:4540-5.

52. Feschotte C, Pritham EJ. Non-mammalian c-integrases are encoded by giant transposable elements. Trends Genet. 2005;21:551-2.

53. Flavell AJ, Ruby SW, Toole JJ, Roberts BE, Rubin GM. Translation and developmental regulation of RNA encoded by the eukaryotic transposable element copia. Proc. Natl. Acad. Sci. United States Am. 1980;77:7107-11.

54. Johns MA, Mottinger J, Freeling M. A low copy number, copia-like transposon in maize. EMBO J. 1985;4:1093-101.

55. Peterson-Burch BD, Voytas DF. Genes of the Pseudoviridae (Ty1/copia Retrotransposons). Mol. Biol. Evol. 2002;19:1832-45.

56. Seberg O, Petersen G. A unified classification system for eukaryotic transposable elements should reflect their phylogeny. Nat. Rev. Genet. 2009;10:276.

57. Lee S-I, Kim N-S. Transposable elements and genome size variations in plants. Genomic Informatics. 2014;12:87-97.

58. Sequencing Consortium THG. Insights into social insects from the genome of the honeybee Apis mellifera. Nature. 2006;444:931-49.

59. Schrader L, Kim JW, Ence D, Zimin A, Klein A, Wyschetzki K, et al. Transposable element islands facilitate adaptation to novel environments in an invasive species. Nat. Commun. 2014;5:5495.

60. Bonasio R, Zhang G, Ye C, Mutti NS, Fang X, Qin N, et al. Genomic Comparison of the Ants Camponotus floridanus and Harpegnathos saltator. Science. 2010;329:1068-71.

61. Hoen DR, Hickey G, Bourque G, Casacuberta J, Cordaux R, Feschotte C, et al. A call for benchmarking transposable element annotation methods. Mob. DNA. 2015;6:13.

62. Bargues N, Lerat E. Evolutionary history of LTR-retrotransposons among 20 Drosophila species. Mob. DNA. 2017;8:7

63. Heringer P, Dias GB, GCS K. A Horizontally Transferred Autonomous Helitron Became a Full Polydnavirus Segment in. G3. 2017;7:3925-35.

64. Coates BS. Horizontal transfer of a non-autonomous Helitron among insect and viral genomes. BMC Genomic-. 2015;16:137.

65. Bao W, Jurka J. Homologues of bacterial TnpB_IS605 are widespread in diverse eukaryotic transposable elements. Mob. DNA. 2013;4:12.

66. Haapa-Paananen S, Wahlberg N, Savilahti H. Phylogenetic analysis of Maverick/Polinton giant transposons across organisms. Mol. phylogenetics Evol. 2014;78:271-4

67. Pritham EJ, Putliwala T, Feschotte C. Mavericks, a novel class of giant transposable elements widespread in eukaryotes and related to DNA viruses. Gene. 2006;390:3-17.

68. Wang B, Geng X-Z, Ma L-B, Cook JM, Wang R-W. A trophic cascade induced by predatory ants in a fig-fig wasp mutualism. J. Anim. Ecol. 2014;83:1149-57.

69. Pérez-Lachaud G, Bartolo-Reyes JC, Quiroa-Montalván CM, Cruz-López L, Lenoir A, Lachaud J-P. How to escape from the host nest: imperfect chemical mimicry in eucharitid parasitoids and exploitation of the ants' hygienic behavior. J. Insect Physiol. 2015;75:63-72.

70. Malcicka M, Bezemer TM, Visser B, Bloemberg M, Snart CJP, Hardy ICW, et al. Multi-trait mimicry of ants by a parasitoid wasp. Sci. reports. 2015:5:8043. 